remifentanil
renal colic
research
residence type
residency education
resident duty hours
resident trainee
resident
resource utilization
respiratory distress
resuscitation
resuscitation guidelines
return of spontaneous circ
reveal
risk Scale
routine investigation
rural
S
safety
satisfaction
screening tools
search engine
sedation
self-reporting
seniors
sepsis
shiftwork
shock
shoulder dislocation
signs and symptoms
simulation

OP32
P006, P007, P050, P085
P103
P030
OP11, P018
LO25, P036, P037
MP37
P070
LO51
P022, P023
OP21, OP28
LO28
P064
OP38
P013
OP20, OP31, P107

LO21, LO25, MP29

P115
MP12, P076
P086

MP23, P024
OP22

MP21, P029

OP37, P107

MP54

OP25, PL01

LO10

$\mathrm{P} 042$

LO01, LO44, MP11,

MP42, MP43, P062, P070,

P091, P094, P095, P096 slit lamp

smoking cessation

social media

socio-economic status

specialist consultation

spinal cord injury

STEMI

stroke

stroke prevention clinic

subarachnoid hemorrhage

superficial thrombophlebitis

suprventricular tachycardia

surgical emergencies

survival

suspected neck injury

symptoms

systematic Review

PP20, P034
LO38, MP41, MP46
P116
OP35
MP36

O03, MP06, OP10, OP13
O04, LO05, LO06, P049
MP28
PL02, LO14, LO34
MP61
P039
MP14
OP19, OP20
OP16
P064
Oia

T

tachycardia

tactical emergency medicine

targeted temperature management

teaching

team error

teamwork

technology

telemetry

therapeutic hypothermia

throughput

tiered response

tongue

transesophageal

transfer

transfusion
MP07

MP44

LO26

P056, P118

$\mathrm{P} 051$

MP26

OP09

MP59

LO28

MP10, MP13

P033

P090

LO20

MP57, PL03

P101, P113 transient ischemic attack

MP28

transport

MP58

trauma

MP01, MP27, MP58, P045, P051, P065, P068, P069,

P073, P099, P101

trauma care organization MP57, PL03

treatment MP61, MP62, P032, P066

tremor

triage

$\mathrm{P} 016$

triage nurse

triage physician

trigger tool

TRISS

LO45, MP60, OP06,

OP14, OP34

LO12

MP13

PL04

$\mathbf{U}$

ultrasound

LO15, LO16, LO17, LO21, MP47, MP55, MP65, OP07, OP25, P012, P052, P092, P094, P095

undergraduate medical education $\quad$ LO22 urinary tract infection $\quad$ OP36, P011 utilization LO18, MP24, MP38, MP40, P054

V

venous thromboembolism MP61, P066

volume surge LO46

volunteer

P104

W

waiting times

weight estimation

welfare

work patterns
LO50

OP31

OP03

LO48

\title{
CAEP/ACMU 2014 Scientific Abstracts Author Index
}

\begin{tabular}{lrl} 
AUTHOR & ABSTRACT No. & Argintaru N \\
\cline { 2 - 3 } A & & $\begin{array}{l}\text { Armstrong A } \\
\text { Armstrong S }\end{array}$ \\
Aarabi P & P016 & Arntfield R \\
Aaron S & OP38 & \\
Abramson BL & OP21 & Arthur AO \\
Abu-Laban RB & MP31 & Assoignon L \\
Adamson M & LO45 & Athaide V \\
Adekoya D & P032 & Atkinson PR \\
Agaybi S & MP09 & \\
Ahmed A & P001 & Atzema CL \\
Ahmed K & P002 & Aufderheide TP \\
Ahmed S & P102 & Austin E \\
Aklilu F & MP25 & Austin M \\
A1 Juma S & P064 & Austin PC \\
Al Kashmiri A & P068 & Azad A \\
Al-Attar M & P097 & Azaj A \\
Albrecht L & LO36 & Azazh A \\
Ali H & P030 \\
Ali S & OP34, P115 & B \\
Allen RA & P003 & Bailey B \\
Alqrashi W & OP30, P004 & Baker L \\
Alzahrani H & P005 & Bandiera G \\
Andruchow J & MP14 & Bank I \\
Archambault PM & P007 & Bardua DJ \\
Archer B & &
\end{tabular}

$\begin{aligned} \text { P006, P085 } & \text { Barratt L } \\ \text { LO29 } & \text { Bastian J } \\ \text { LO29 } & \text { Beaudry P } \\ \text { LO20, LO22, MP47, } & \text { Bednarczyk JM } \\ \text { P012, P050 } & \text { Bégin F } \\ \text { LO02, MP01, MP44, } & \text { Bell R } \\ \text { P039, P040, P041, P042 } & \text { Benjamin S } \\ \text { P072 } & \text { Berman S } \\ \text { MP19 } & \text { Bernklau R } \\ \text { LO48, MP17, P007, } & \text { Betz M } \\ \text { P019, P069, P073, PL01 } & \text { Bhanich Supapol W } \\ \text { LO04, P021 } & \text { Bhatti JA } \\ \text { OP28 } & \text { Bhimani M } \\ \text { MP43 } & \text { Bhutani M } \\ \text { OP22 } & \text { Bidlake E } \\ \text { LO04, P021 } & \text { Bienkowski A } \\ \text { MP25 } & \text { Bierman AS } \\ \text { P067 } & \text { Bilodeau A } \\ \text { MP43, P065, P102 } & \text { Birnie D } \\ & \text { Bishnoi A } \\ & \text { Blackie B } \\ \text { LO29 } & \text { Blake DF } \\ \text { MP48 } & \text { Blake GD } \\ \text { LO47, P058 } & \text { Bloom GA } \\ \text { OP07, P008, P080 } & \text { Blouin D } \\ \text { LO52 } & \text { Bohrer M } \\ \text { OP15 } & \text { Boissy P }\end{aligned}$

OP37, P058, P107

P059

MP59

LO41

MP03

$\mathrm{LO} 23$

P069, P073

P065, P067

$\mathrm{P} 112$

OP06

MP13

MP25

P006, P085

P089

P026

MP04

OP21

LO38

MP23, P024

P118

P075

P009

P009

P064

LO38

OP12

OP16 
Bonkowski KN

Borgundvaag B

Boudreau M

Boulay J

Bourdon E

Bourque CJ

Boutis K

Bowen J

Bradley J

Brine M

Brison RJ

Bromberg S

Brooks SC

Brown N

Brown R

Bryan JM

Buchanan IM

Buhariwalla $\mathrm{H}$

Bullard M

Burbridge $\mathrm{B}$

Burge F

Burgess R

Burns BD

Bussières $\mathrm{S}$

Butler M

Byers A

Byrne C

C

Cadogan M

Cagaanan R

Cajee I

Calder LA

Calder-Sprackman S

Callaham M

Campbell CL

Campbell SG

Campbell S

Caners K

Canuel A

Carrigan S

Carter A

Carter LV

Carver SM

Certain M

Chabot P

Chalut D

Chan K

Chan TM

Chandra K

Charest-Bossé M

Charette M

Chauny J

Chen $\mathrm{H}$

Chen J

Chen N

Chen S

Cheng A

Chenkin J

Cheskes S

Chetram R

Cheung J

Cheung K

Chipperfield K

Cho DD

$\begin{aligned} & \text { P010 } \text { Choi S } \\ & \text { LO17, MP23, MP32, } \text { Christenson J } \\ & \text { OP38, P016, P024 } \text { Chung T } \\ & \text { OP15 } \text { Clark D } \\ & \text { OP16 } \text { Cleiman P } \\ & \text { MP68 } \text { Clement CM } \\ & \text { OP22 } \text { Clemente M } \\ & \text { P103, P104 } \text { Clinkard D } \\ & \text { OP13 } \text { Cohen L } \\ & \text { LO21 } \text { Common MR } \\ & \text { P011 } \text { Compeau S } \\ & \text { P016 } \text { Cook J } \\ & \text { LO26 } \text { Cookson C } \\ & \text { MP23, OP38, P024 M } \\ & \text { MP68 } \text { Correa JA } \\ & \text { MP05 } \text { Cory B } \\ & \text { MP58 } \\ & \text { LO27 } \text { Courchesne CE } \\ & \text { OP14, P061 } \text { Craig W }\end{aligned}$

Craig W

Crevier L

Crick K

Croskerry P

Cross E

Crudden J

$\begin{array}{ll}\text { LO31, MP64, MP70, } & \text { Cruise S } \\ \text { 7, OP27, P013, P045, } & \text { Cummings G }\end{array}$

$\mathrm{P} 046, \mathrm{P} 047, \mathrm{P} 048$

OP19, OP20

LO22, MP47, P012

Curry G

Curtis S

Curtis SJ

Cwinn A

MP46

LO24

MP64, OP27, P013

LO24, LO43, MP04,

MP26, OP22, OP38, P026

LO24, P027

MP49

MP64, OP27, P013

MP64, OP27, P013

LO11, P072

OP13

$\mathrm{P} 025$

$\mathrm{P} 015$

MP68, OP12, OP15, P015

$\mathrm{P} 028$

LO17, P016

$\mathrm{P} 025$

OP18

MP27

OP30

LO07, MP41, MP49,

OP11, P018, P086, P108

P019 Desjardins M

OP16 Deveau B

OP23 Devlin G

LO30, MP22, MP53,

MP59, OP02, OP29

MP62

P020

MP55

MP61

MP10, LO47

LO09

OP19, OP20, OP21, OP28

LO11, LO50, MP34,

MP50, P077, P078

MP28

MP20, P034

P113

P021
Dagnone J

ain $\mathrm{C}$

Dainty KN

Daly-Grafstein B

Daoust R

Davidson A

Davison C

de Montigny L

de Moor M

De Sousa $\mathrm{N}$

Deady B

DeBruyne B

Delaney J

Delaney SJ

Demendonca B

Dempster L

Devlin SM

Diegelmann L

Dolansky G

Dong K

Dorian $\mathrm{P}$

Douglass ER

Dowdell T

Dreyer J

Dubrovsky A

Ducas J

Ducas RA

Duggan LV

Dukelow A

Dukundane D

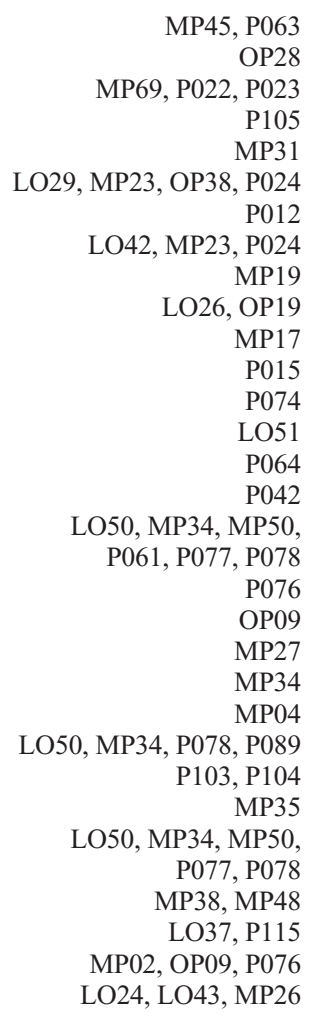

Duncan S

Dupuis G

LO28

$\mathbf{E}$

Eagles D

Earle RK

Edmonds M

Emond M

Eppler J

Erdogan M

Erskine M

Ertel E

Ertel M

Evans J

F

Fabian JC P032

Falconer C

Feenstra S

Feldman M

Ferguson J

Ferlatte N

Fichtinger G

Field S

Filiatrault L

Filipowska C

Fingrut W

Finningley A

Fisher R

Fitzpatrick E

Fitzpatrick S

Fleet R

Forster A

Fountain RB

Fowler R

Francis L

Frank J

Frank JR

MP39, MP59

LO11, LO50, MP34,

MP50, P077, P078

LO42, LO44

MP07

LO46, P043

P019

P028

LO16, P052

$\mathrm{MP02}$

MP25, P064

OP16

OP05

PL04

LO37

MP06

P105

OP28

PL01

$\mathrm{P} 027$

P057, P115

LO26, MP23, OP21,

OP28, $\mathrm{P} 024$

LO16, P052

LO47

LO23, OP36

OP07

MP63

MP63

P028

MP09, P106

LO16, P052

LO06, OP02

MP12, MP23, P024

P083

P060

LO10, LO13, LO30, MP15,

MP21, MP39, MP53, MP57,

OP18, OP29, P029,

PL02, PL03
P030, P082

LO31

P031

P082

$\mathrm{P} 082$

$\mathrm{P} 038$

P113

MP05, OP05, P033

OP31

$\mathrm{P} 025$

LO42

MP64, OP27, P013

LO12

MP42, P070

$\mathrm{P} 034$

OP31

OP34, P075

LO51

LO06, LO38, MP03, MP65, OP02

LO24, OP38

LO38

OP21

P026, P087

LO25, LO43, MP42,

MP45, P036, P037,

P063, P070, P118

Fraser J

French J

Friedman SM

Froese P

Frost DM

Fuller D

Fung W

Fuss J

019, P069

P069

MP31

P101

MP13

OP01, OP04

MP28

P002

G

Gagnon S

Gandhi R

Garniss D

Gatien M

Gaucher N

Gaudio M

Gaunt K

Gauthier J

Gauvin F

Genzel R

Ghag D

Giguère J

Gilbert D

Given LM

Goel G 


\begin{tabular}{|c|c|c|c|c|c|}
\hline Goodman SG & $\mathrm{LO} 26$ & Jepson M & P057 & Langmann $\mathrm{C}$ & MP13 \\
\hline Gorman LJ & LO25, P036, P037 & Jervis A & MP60 & Larocque $\mathrm{N}$ & LO24 \\
\hline Gouin S & LO37, MP27 & Ji D & MP62 & Lavoie A & MP57, PL03 \\
\hline Goulard D & MP71 & Johnson C & P005 & Law S & P066 \\
\hline Gould J & $\mathrm{P} 015$ & Johnson D & P097 & Le May M & LO28 \\
\hline \multirow[t]{2}{*}{ Grafstein E } & LO46, LO51, MP61, & Jolley J & P058 & Le Sage N & LO30, MP21, MP53, MP57, \\
\hline & MP62, P043, P066 & Jones S & MP60, P059 & & OP18, OP29, P029, PL03 \\
\hline Graham M & OP24, OP26 & Joshi N & P108 & Lebel D & LO37 \\
\hline Gravel J & MP27, OP33, P025 & Joubert G & MP55, P011 & Lebel K & OP16 \\
\hline \multirow[t]{2}{*}{ Green R } & LO31, MP70, P045, P046, & Juste L & MP07 & Lee C & MP08 \\
\hline & P047, P048, P101 & Justice W & MP44 & Lee JS & LO13, MP15, MP21, MP39, \\
\hline Grenier D & P029 & & & & $\mathrm{P} 029, \mathrm{P} 044$ \\
\hline Grewal K & LO04 & $\mathbf{K}$ & & Lee WW & LO12 \\
\hline Grierson R & LO52 & Kabaroff A & MP69, P022, P023 & Légaré F & LO38, MP65, OP02 \\
\hline Griesdale DE & P028 & Kadhim-Saleh A & LO18, MP24, MP40 & Léger P & MP65 \\
\hline Grushka D & P006, P085 & Kahan M & P016 & Leigh $\mathrm{R}$ & P089 \\
\hline Guttmann A & LO23 & Kang H & P060 & LeMay S & LO37, OP33, P025 \\
\hline Guy AS & & Karpov A & PL04 & Leroux Y & MP06 \\
\hline $\mathbf{H}$ & & Karreman E & OP08, P010, P110 & Lesage $\mathrm{S}$ & LO39, MP11 \\
\hline Haggerty J & OP02 & Kavsak P & MP66 & Lesiuk H & PL02 \\
\hline Haligua A & P026 & Kawano T & MP16 & Lessard J & MP22 \\
\hline Hall AK & LO44 & Kedia R & LO03 & Letson C & P039 \\
\hline Hames H & LO22, LO53 & Kempinska A & OP07 & Leung AP & P082 \\
\hline Hanel E & P109 & Khalil E & P008 & Leung A & MP55 \\
\hline Hanson A & OP09 & Khan M & LO14, LO34 & Levin $\mathrm{H}$ & MP54 \\
\hline Harkema S & MP36 & Kimura T & MP16 & Levy A & LO39, MP11 \\
\hline Harris DR & LO05, MP61, MP62, P049, P066 & King D & LO47 & Levy P & P105 \\
\hline Hartling L & P097 & King $\mathrm{H}$ & P073 & Lewell M & P074 \\
\hline Hassani B & P050 & King M & LO49, P079 & Lewis D & PL01 \\
\hline Hayashi $\mathrm{H}$ & MP16 & Kircher J & LO36, OP34, P115 & Lewis EC & P038 \\
\hline Hayes C & OP37, P107 & Kirkham A & P057 & Liew A & LO21 \\
\hline Hébert D & MP03 & Kirkland SW & LO11, LO50, MP34, & Lim R & MP55 \\
\hline Hecht N & LO15 & & MP50, P061, P072, & Lima M & MP33 \\
\hline Hefferon D & OP05 & & P077, P078 & Lin D & MP28, P109 \\
\hline Heipel H & P051 & Kisilewicz M & OP32 & Lin $\mathrm{M}$ & MP41, MP46, MP49, \\
\hline Henwood PC & LO16, P052 & Klassen T & MP71, OP09, P097 & Lingard L & LO40 \\
\hline Hicks C & P051 & Klingel M & LO40, LO45, MP08, & Lipkus M & P106 \\
\hline Higgins L & $\mathrm{P} 040, \mathrm{P} 041$ & & MP09, MP35, MP56, OP36, & Liss $\mathrm{K}$ & MP69, P022, P023 \\
\hline Hill S & MP66 & & P001, P002, P011, P020, P060, & Liteplo AS & LO16, P052 \\
\hline Hogue C & $\mathrm{P} 025$ & & P068, P100, P106 & Little B & P110 \\
\hline Hohl CM & MP19, PL02, PL04 & Knisley L & MP71 & Liu N & LO12 \\
\hline Holden M & LO42 & Kolesnik J & MP28 & Liu YS & OP35, P071 \\
\hline Holder P & $\mathrm{P} 040, \mathrm{P} 041$ & Kontos MC & OP28 & Lobay K & OP14 \\
\hline Holroyd BR & OP24, OP26 & Kortko J & OP22 & Lockwood J & P067 \\
\hline \multirow[t]{2}{*}{ Holroyd B } & LO50, MP34, MP50, & Kovacs GJ & P062 & Logue E & OP09 \\
\hline & P077, P078 & Krassioukov A & MP36 & Lonergan $\mathrm{K}$ & MP48 \\
\hline Howes D & P088 & Krause C & OP08 & Longeway M & P081 \\
\hline Howlett M & LO48, MP17 & Krebs E & P052 & Loubani E & $\mathrm{P} 100$ \\
\hline Huang D & MP36 & Kruck C & MP71 & Loubani O & MP06 \\
\hline Huard V & MP59 & Kulkarni G & LO04 & Loubani $\mathrm{T}$ & P068 \\
\hline Hunchak, C & MP32 & Kwok ES & LO43, MP30, P063 & Lowe M & LO29 \\
\hline Hunter JJ & MP32 & & & $\mathrm{Lu} \mathrm{H}$ & LO04 \\
\hline \multirow[t]{2}{*}{ Hurley B } & LO15 & $\mathbf{L}$ & & Lukanovic D & P069 \\
\hline & & Laidley I & OP10 & Lund A & LO33, P083 \\
\hline I & & Lalonde K & $\mathrm{LO} 21$ & Lynch TP & MP54 \\
\hline Ilyas F & P116 & Lam TV & LO33 & Lyster K & P110 \\
\hline Imrie $\mathrm{K}$ & LO25, P036, P037 & Lamfookon $\mathrm{C}$ & P064 & & \\
\hline \multirow[t]{3}{*}{ Innes $\mathrm{G}$} & MP18, MP38, MP48, & Lamprecht $\mathrm{H}$ & PL01 & $\mathbf{M}$ & \\
\hline & $\mathrm{P} 054, \mathrm{P} 055$ & Lanctôt J & OP25 & Mack L & MP14 \\
\hline & & Landes M & MP43, P065, P067, P102 & Mackenzie DC & LO16, $\mathrm{P} 052$ \\
\hline $\mathbf{J}$ & & Lang C & MP61 & MacKinnon D & LO47, OP37, P058, \\
\hline Jabbour M & P097 & Lang E & MP14, MP15, MP18, & & P099, P107 \\
\hline Jamani Lund S & P083 & & MP21, MP23, MP38, MP39, & MacLellan J & LO36 \\
\hline Jarvis C & P056 & & MP48, MP60, MP71, OP35, & MacPhee S & OP17 \\
\hline Jean-Frédéric L & OP02 & & OP39, P003, P024, P049, P054, & Magee K & MP23, MP64, OP27, \\
\hline Jelic $\mathrm{T}$ & MP63 & & P055, P059, P071, P089 & & $\mathrm{P} 013, \mathrm{P} 024$ \\
\hline \multirow[t]{2}{*}{ Jensen JL } & MP04, MP06, MP68, & Langhan $\mathrm{T}$ & MP23, P024 & Mah H & $\mathrm{P} 113$ \\
\hline & OP12, OP15, P015 & Langlais J & LO29 & Mainprize D & P012 \\
\hline
\end{tabular}




Majumdar SR
Mall O
Maloney J
Maltais-Giguère J
Mann M
Marcantonio R
Marshall EG
Martin G
Martineau M
Martinez M
Maskalyk J
Massaro P
Masse B
Massie K
Mastoras G
Matchett S
Maunder RG
Mayich J
Mazer C
McCabe C
McConnell AH
McGowan M

McNeil G

McNitt W

McRae A

McVey J

McWilliam M

Melady D

Mercuur L

Meshkat N

Messenger DW

Meyers C

Migneault D

Miller J

Miller P

Miller TL

Millington S

Milne J

Milne K

Minhas KS

Moe G

Moe J

Mohabir IM

Mok C

Moncrieff K

Mondoux SE

Montazeri R

Moore J

Moore L

Moore S

Moreau K

Morrin L

Morrison LJ

Moss M

Mullenger RE

Munkley D

Murnaghan A

Murray AF

\begin{tabular}{|c|c|}
\hline P089 & Murray M \\
\hline P109 & Murry W \\
\hline OP22 & Myslik F \\
\hline MP65 & \\
\hline P050 & $\mathbf{N}$ \\
\hline $\mathrm{LO} 27$ & Nagatu E \\
\hline OP15 & Nam J \\
\hline P058 & Naranian $\mathrm{T}$ \\
\hline $\mathrm{P} 025$ & Nauss M \\
\hline MP01 & Nayar A \\
\hline MP43, P067 & Nazif A \\
\hline P007 & Nesbitt R \\
\hline MP27 & Neto G \\
\hline MP44 & Neveu X \\
\hline MP26 & Newton A \\
\hline MP17 & Newton S \\
\hline MP32 & $\mathrm{Ng} \mathrm{M}$ \\
\hline P073 & $\mathrm{Ng} \mathrm{P}$ \\
\hline P105 & $\mathrm{Ng} \mathrm{V}$ \\
\hline P089 & Nguyen L \\
\hline MP42, P070 & Nikel T \\
\hline LO19, LO47, OP37, & \\
\hline P051, P058, P099, & Ninkovich $\mathrm{S}$ \\
\hline $\mathrm{P} 105, \mathrm{P} 107$ & Nixon $\mathrm{K}$ \\
\hline LO42 & Noble V \\
\hline LO32 & Norouzi N \\
\hline LO22, MP47, P012 & Noseworthy R \\
\hline LO20, LO35, LO45, & Nugus $\mathrm{P}$ \\
\hline LO53, MP08, MP09, MP35, & \\
\hline MP42, MP56, OP36, P001, & $\mathbf{O}$ \\
\hline P002, P011, P020, P050, P060, & O’Reilly D \\
\hline P068, P070, P100, P106 & Obaid A \\
\hline LO44 & Odenbach $\mathrm{J}$ \\
\hline P042 & Odorizzi S \\
\hline MP14, MP18, MP23, & Ohle R \\
\hline MP38, MP48, OP35, & Okrainec A \\
\hline OP38, P024, P054, & Oleksak K \\
\hline P055, P059, P071 & Oliveria $\mathrm{J}$ \\
\hline MP06, MP68, OP15 & Olszynski P \\
\hline $\mathrm{P} 074$ & Olszynski PA \\
\hline LO08 & Ornato JP \\
\hline MP38, MP48 & Osborne C \\
\hline MP43 & Ospina MB \\
\hline LO42 & \\
\hline LO36 & Otal D \\
\hline LO41 & Ouellet M \\
\hline LO29 & Ouellet P \\
\hline MP43 & Ouimet M \\
\hline P028 & Ovens HJ \\
\hline P005 & Oxciano $\mathrm{P}$ \\
\hline PL01 & \\
\hline P006, P085 & $\mathbf{P}$ \\
\hline P028 & Pace J \\
\hline P105 & Packer $\mathrm{N}$ \\
\hline $\mathrm{P} 072$ & Pageau P \\
\hline MP31 & Panditha C \\
\hline PL04 & Paquet J \\
\hline MP71 & Paquin $\mathrm{H}$ \\
\hline LO43 & Parcero C \\
\hline P073 & Pardhan A \\
\hline LO28 & Park H \\
\hline MP53, MP57, PL03 & Parker BA \\
\hline LO21, LO44 & Parker S \\
\hline P087 & Parry N \\
\hline OP39 & Parsons J \\
\hline LO26, OP19, OP20, & Parush A \\
\hline OP21, OP28 & Pat A \\
\hline P074 & Patel RJ \\
\hline $\mathrm{P} 075$ & Paterson Q \\
\hline OP10 & Paterson QS \\
\hline P087 & Paul JE \\
\hline LO16, P052 & Pawsey R \\
\hline
\end{tabular}

Murray M

Myslik F

Nam J

Nauss M

Nayar A

Nesbitt R

Neto G

Newton A

Newton S

$\mathrm{Ng} \mathrm{M}$

$\mathrm{Ng} \mathrm{V}$

Nguyen L

Ninkovich S

Nixon K

Noseworthy R

O'Reilly D

Obaid A

Odorizzi S

Ohle R

(

Oliveria J

zynski $P$

Ornato JP

Osborne C

Otal D

Ouellet M

Ovens HJ

Oxciano $\mathrm{P}$

Pace J

Pageau $\mathrm{P}$

Panditha C

Pardhan A

Park H

Parker S

Parry N

Parush A

Patel RJ

Paterson Q

\begin{tabular}{|c|c|c|}
\hline P062 & Peddle B & MP56 \\
\hline P040, P041 & Peddle M & MP08 \\
\hline \multirow[t]{3}{*}{ P074 } & Peet HE & $\mathrm{P} 112$ \\
\hline & Peirce $\mathrm{M}$ & P074 \\
\hline & Penciner R & P084 \\
\hline OP37, P058, P107 & Perry JJ & LO13, LO14, LO18, \\
\hline OP13 & & LO32, LO34, MP15, \\
\hline P103, P104 & & MP21, MP23, MP24, \\
\hline MP51 & & MP39, MP40, OP38, \\
\hline P081 & & P024, P032, PL02 \\
\hline LO5 1 & Pettersen G & LO39, MP11 \\
\hline $\mathrm{P} 010$ & Phillips DD & OP36 \\
\hline OP30, P004 & Pickett TA & $\mathrm{OP} 03$ \\
\hline MP15, MP39, MP53, OP18 & Pickett W & $\mathrm{LO} 44$ \\
\hline OP34, P076, P115 & Piette & MP65 \\
\hline OP17 & Plant J & LO06, MP65 \\
\hline OP35, P071 & Plint A & OP34, P027 \\
\hline LO19 & Poitras J & LO06, LO38, MP65, OP02 \\
\hline P020 & Polson $\mathrm{K}$ & P113 \\
\hline P080 & Poonai N & MP54, MP55 \\
\hline LO11, LO50, MP34, & Porter SC & MP33 \\
\hline MP50, P077, P078 & Posselwhite $\mathrm{K}$ & LO29 \\
\hline LO45 & Poulin C & $\mathrm{LO} 28$ \\
\hline P058, P099 & Poureslami I & LO05 \\
\hline LO16, P052 & Pozgay A & $\mathrm{P} 035$ \\
\hline $\mathrm{P} 016$ & Preyra I & LO07, MP13 \\
\hline $\mathrm{P} 028$ & Price L & P060 \\
\hline \multirow[t]{3}{*}{ P080 } & Principi T & MP33 \\
\hline & Proulx M & MP07 \\
\hline & Purdy E & LO41 \\
\hline OP13 & Pytka S & OP35, P071 \\
\hline P068 & & \\
\hline P076 & $\mathbf{R}$ & \\
\hline MP35 & Rac VE & OP 21, OP 28 \\
\hline LO32 & Racz E & OP21 \\
\hline MP43 & Radecki RP & MP49 \\
\hline MP31 & Rafo N & P006, P085 \\
\hline P103, P104 & Rahimpour M & MP26 \\
\hline PL01 & Rahman A & $\mathrm{MP} 02$ \\
\hline LO01 & Raine $\mathrm{T}$ & P086 \\
\hline OP28 & Rashidi A & LO12 \\
\hline $\mathrm{LO} 28$ & Reardon M & LO15 \\
\hline LO49, LO50, MP50, & Redfearn D & MP23, P024 \\
\hline P077, P078, P079 & Reed A & OP22 \\
\hline MP12 & Reiter K & MP31 \\
\hline MP53 & Rempell JS & LO16, P052 \\
\hline OP18 & Renouf T & P114 \\
\hline $\mathrm{OP} 02$ & Reynolds SC & P028 \\
\hline MP32 & Rezaie SR & MP49 \\
\hline \multirow[t]{3}{*}{ MP36 } & Rich T & MP48 \\
\hline & Richardson C & MP56 \\
\hline & Riggan M & LO53 \\
\hline LO20 & Robertson $\mathrm{T}$ & P087 \\
\hline LO22, MP47, P012, P106 & Robitaille N & LO39, MP11 \\
\hline $\mathrm{LO} 15, \mathrm{LO} 27, \mathrm{LO} 28, \mathrm{P} 005$ & Romano S & MP09 \\
\hline PLO4 & Ronson A & $\mathrm{LO} 25, \mathrm{P} 036, \mathrm{P} 037$ \\
\hline MP22, MP59 & Rose J & MP68 \\
\hline P080 & Rose N & MP19 \\
\hline PL04 & Rose S & MP23, P024 \\
\hline OP11 & Rosenberg $\mathrm{H}$ & OP32 \\
\hline P081 & Ross D & MP07 \\
\hline P082 & Ross JR & LO44, P062 \\
\hline MP01 & Rossoni M & P074 \\
\hline P068 & Rosychuk RJ & OP24, OP26, OP34, \\
\hline OP21 & & P089, P115 \\
\hline MP26 & Roth N & P088 \\
\hline OP35, P071 & Roudsari A & P031 \\
\hline LO03 & Rowe BH & LO11, LO23, LO49, \\
\hline MP46 & & LO50, MP23, MP34, \\
\hline OP01, OP04 & & MP50, MP69, OP24, \\
\hline P083 & & OP26, OP38, P022, P023, P \\
\hline P007 & & 024, P061, P072, P077, \\
\hline
\end{tabular}


Roy $\mathrm{H}$

Roy M

Rubin M

Ruddy K

Ruddy M

Rulisa S

Ryan E

S

Sadoway R

Sahlas D

Salvalaggio G

Samoraj K

Sampsel K

Sanchez M

Sanders JL

Sandeski R

Sandhanwalia SK

Sangha G

Sansregret A

Sarrazin J

Saude EJ

Savage DW

Sbrocca N

Scales DC

Scheuermeyer FX

Schlamp RS

Schull M

Scott S

Scoville J

Seabrook J

Sedran R

Segal E

Sell E

Senthilselvan A

Shabestari O

Shah A

Shah K

Shaw A

Shea J

Shearer PW

Shepherd L

Sherbino J

Shortt C

Shrier

Shy BD

Silver A

Simard R

Simard-Racine G

Sinclair D

Sinclair J

Sinclair JE

Sinha SK

Sirois M

Sivilotti M

Skitch S

Slomer A

Slutsky A

Smith KE

Smith SM

Smith V

Sne N

Sohmer B

Sookram S

Sowers N

Spence J

Stachura MR

Stander M

Startup C

Steadman PE
P078, P079, P089

MP52

MP22, MP59

MP29, P090, P091,

P092, P093, P094, P095

LO29

LO16, P052

$\mathrm{P} 030$

OP31

MP28

$\mathrm{P} 057$

MP67

MP45, P063

$\mathrm{P} 025$

MP46, P108

$\mathrm{P} 062$

MP67

MP54

LO39, MP11

P102

P003

P096

LO45

LO26, OP21

LO51

P112

$\mathrm{LO} 23$

P097

P019

MP54

MP35

MP07, OP16

$\mathrm{P} 038$

LO49, P079

$\mathrm{P} 03$

P011, P050

$\mathrm{LO} 03$

MP63

MP17

$\mathrm{LO} 03$

LO40

LO07, LO41, MP13, P018

MP66

OP16

$\mathrm{LO} 03$

OP20

$\mathrm{P} 044$

$\mathrm{OP} 02$

MP71

OP23

OP22

MP12

LO13, MP15, MP21, MP39

LO14, LO23, LO34, PL02

P098

P099

OP21

OP14, P057

OP08

P100

MP58

MP26

MP02

P101

$\mathrm{P} 058$

P102

PL01

LO46, P043

P103, P104
Steeg JB

Steele RJ

Steinhart B

Stempien J

Stenstrom R

Stephenson F

Stewart L

Stewart M

Stickland MK

Stiell IG

Stitt D

Stone A

Strauss BJ

Strayer RJ

Stukel T

Sullivan E

Sullivan K

Sutherland F

Sutherland J

Swan J

Szulewski A

T

Ta R

Taber S

Talbot J

Taljaard M

Tambakis M

Tamura $\mathrm{T}$

Tanguay AB

Tavares W

Tay JK

Taylor L

Taylor M

Taylor S

Tenenbein M

Thanh J

Theakston K

Theoret J

Thiruganasambandamoorthy V LO15, P005

Thoma B

Thomas AJ

Thomas D

Thomas SH

Thompson D

Thorne S

Thurger L

Tounkara F

Traboulsi M

Tran M

Tran S

Travers A

Treasurywala K

Trivedi S

Trottier ED

Tsai A

Tsai J

Turgeon $\mathrm{P}$

Turner L

Turris SA

Tyberg J

$\mathbf{U}$

Ungii T

LO46, P043

LO49, P079

LO21, LO24, LO29,

MP12, MP23, OP23, OP28,

OP30, OP38, P004,

P024, PL02

LO15
MP35

P106

LO03

OP01, OP04

LO52

MP14

PL02

LO25, P036, P037

LO48

LO18, MP24, MP40, P032

LO45

MP69, P022, P023

MP03, OP02

MP04

P003
P114

L015

OP37, P107

LO52

$\mathrm{LO} 38$

P001

LO41, MP41, MP46,

MP49, P086, P108

OP28
OP34

LO02, MP01, MP44,

P039, P040, P041, P042

LO22, MP47, P012

$\mathrm{LO} 29$
$\mathrm{P} 116$

L006

OP39

P032

P109

MP04, MP06, MP68,

OP15, P015

MP28

OP06, P110

OP33, P025

MP20

MP20

MP53

MP05, P033

$\mathrm{P} 083$

OP05

V

Vacon C

Vaillancourt C

Vaillancourt S

Vair B

Valani R

Valois M

van de Belt TH

Van Den Broek K

van Diepen K

Vanberkel P

Vandenberghe $\mathrm{C}$

Vandenberghe $\mathrm{H}$

Vandermeer B

Vanier L

Varner C

Varpio L

Venugopal R

Vermeulen MJ

Verreault R

Vickerman SV

Vieira A

Villa-Roel C

Vincent M

Visintini S

Voaklander B

oaklander D

Vu EN

W

Wagg J

Waisberg L

Walker M

Walker T

Wand RT

Wang A

Wang D

Wang J

Warren Z

Weaver B

Weingarten L 
Résumés scientifique 2014 — Index des auteurs

$\begin{array}{lrlrlr}\text { Wu A } & \text { P001 } & \text { Yang J } & \text { MP51 } & \text { Z } \\ \text { Wyse G } & \text { MP23, P024 } & \text { Yau L } & \text { LO12 } & \text { Zaver F } & \text { MP37 } \\ & & \text { Yeoman A } & \text { MP17 } & \text { Zemek R } & \text { P027 } \\ \text { Y } & & \text { Yiu SH } & \text { P117 } & \text { Zhan C } & \text { OP19, OP20 } \\ \text { Yan D } & \text { MP36 } & \text { Young M } & \text { P008, P080 } & \text { Zibrowski E } & \text { LO40 } \\ \text { Yan J } & \text { LO35 } & \text { Yu E } & \text { PL04 } & \text { Zlepnig J } & \text { LO24 } \\ \text { Yan W } & \text { MP47 } & \text { Yusuf O } & \text { P118 } & \text { Zurba J } & \text { P028 }\end{array}$

